\title{
Impact of a nutrition/educational program in a group of type 2 diabetes patients, already involved in a wider "Group Care" plan and not achieving complete target: an observational study
}

\author{
Anna Comba, Alessandra Ardizzone, Laura Leto, Mauro Feola \\ Cardiovascular Rehabilitation - Heart Failure Unit, Ospedale SS. Trinità, Fossano, Cuneo, Italy
}

\begin{abstract}
Type 2 diabetes is one of the most frequent chronic degenerative diseases in the world (5.4\% in Italy); it has many chronic consequences with a significant impact either on expectation or quality of life. About $80-90 \%$ of type 2 diabetes patients is either overweight or obese, condition that can cause many more negative consequences than in individuals in the same conditions but with normal weight. The aim of the research is to assess if diabetic people who cannot reach the goals of glycemic control, good eating habits and weight loss can eventually obtain, with an individualized care plan, long lasting improvements. This study involved a little group of diabetic patients who did not achieve an optimal therapeutic target.

These patients have been individually followed by a dietician for six months through a program of dietetic counseling and regular controls and at the end we compare the results of another group of diabetic people, involved in the Group Care Plan (but without the support of the dietitian). The nutrition educational program determined a significant weight loss $(-4.62 \mathrm{~kg} v s+3.46 \mathrm{~kg} ; \mathrm{p}<0.05)$, a reduction of waist circumference $(-5.34 \mathrm{~cm} v s+4.15 \mathrm{~cm}$; $\mathrm{p}<0.05)$, an improvement of glycemic control (Hb1Ac: $-0.67 \% v s+0.57 \%$; $\mathrm{p}<0.05$ ), lipidic profile (total cholesterol: $-1 \mathrm{mg} / \mathrm{dl} v s+12.85 \mathrm{mg} / \mathrm{dl}$; $\mathrm{p}<0.05$ ) and perceived quality of life (Test Att 19: 0 subjects vs 6 subjects). An individualized nutrition educational program in patients with type 2 diabetes not achieving a therapeutic target can optimize global care of the disease.
\end{abstract}

Corresponding author: Dr. Anna Comba, Cardiovascular Rehabilitation Heart Failure Unit, Ospedale SS. Trinità, via Ospedale 4, 12045 Fossano (CN), Italy. Tel. $+39.320 .4431987 ;+39.0172 .699365$ - Fax +39.0172 .699364$ E-mail: anna.comba@libero.it; riabilitazionecardio.fossano@asın1.it

Key words: Nutrition; educational program; type 2 diabetes; Group Care.

Received for publication: 10 0ctober 2016

Accepted for publication: 15 March 2017

(C) Copyright A. Comba et al., 2017

Tipografia PI-ME Editrice, Italy

Monaldi Archives for Chest Disease 2017; 87:780

doi: 10.4081/monaldi.2017.780

This article is distributed under the terms of the Creative Commons Attribution Noncommercial License (by-nc 4.0) which permits any noncommercial use, distribution, and reproduction in any medium, provided the original author(s) and source are credited.

\section{Introduction}

It is known that being overweight is a salient factor in the pathogenesis of insulin resistance and that weight loss is a very effective means of improving Glycometabolic control.

In addition, excess weight is associated with an increased prevalence of cardiovascular risk factors, therefore weight control is of great importance in the longterm prevention of complications associated with many chronic diseases, among which type 2 diabetes (T2DM) [1-3].

The numerous studies carried out have allowed researchers to observe how, in patients with T2DM, the most important results, in terms of reduction of total mortality rates and cardiovascular events (over 50\% of cases) have been obtained through intensive intervention aimed at the optimization of all the risk factors involved. Therefore, only a comprehensive approach to the disease, not limited to glucose control, but extended to the different components of cardiovascular risk, may permit a clinically significant reduction in the impact of macroangiopathic complications on mortality and morbidity in diabetes [4-5].

Based on these assumptions, over the last ten years the Laboratory of Clinical Pedagogy of the University of Turin has designed and developed a method of nutrition education called "Care Group" for patients with T2DM, which includes regular meetings where themes regarding the management of the illness are dealt with, together with the control of the medical parameters. A decrease in body weight, together with an increase in HDL cholesterol and glycated hemoglobin stabilization has been observed [6-8].

This educational method is used in many clinics of diabetology in Piedmont including, as from 2008, the diabetes outpatient department at the Hospital "SS. Trinità" of Fossano.

Group Care is a practical educational method, focused on lifestyle management. Furthermore, it permits the analysis of all those aspects which have brought to light how the unresolved issues for some patients have always been weight loss and diet and nutrition management, as well as the false myths surrounding the disease. It becomes, therefore, necessary to select recipients for a more targeted and personalized intervention. In fact, although on one hand it may be insufficient in some cases to merely outline the general guidelines for healthy living, on the other hand, offering a personalized programme to all patients with T2DM would be a depletion of resources, whereas identifying those patients in need of direct intervention and dedicating stricter controls and more intensive care to these individuals would allow for the optimization of resources and the achievement of the therapeutic aims would be facilitated. This remains in accordance with current pharmacological practice, which aims to adapt the therapy to the individual patient. 
The purpose of our trial was to verify if an individual programme with regular checks, the drafting of a personalized dietary plan and the inclusion of sustainable changes produce significant benefits on glycemic control, on metabolic parameters and on the quality of life in a group of T2DM patients not responsive to Group Care.

\section{Materials and Methods}

Twenty-eight patients were selected from among those with T2DM who had been referred to the Diabetes Outpatients department at Fossano Hospital and who were participating in educational meetings on the Group Care model. In particular, those selected were not "target" patients, defined as patients with a BMI (body mass index - BMI) $\geq 26$ and suboptimal glycemic control (glycated hemoglobin - HbAlc) $\geq 7 \mathrm{mg}$ / dl. There was a 6-month follow-up period (from March to December 2014).

Of the 28 patients enrolled, 14 were referred to an outpatient path in which individual scheduled meetings with the dietician were set up, anthropometric parameters were assessed (body weight, waist circumference, BMI calculation), a personalized dietary plan was elaborated and nutritional education with regular checks provided. The food history for each individual was recorded in a food diary and a $24 \mathrm{~h} \mathrm{Di}$ etary recall (or recall of 24 hours, 24HR).

All participants were provided with a diet plan in compliance with the latest scientific evidence available, nutritionally balanced, personalized (according to age, sex, concurrent pathologies and food intolerance), with a reduction in calorie intake with respect to habitual levels in order to favour weight loss. None of the diet plans foresees an intake of less than $1400 \mathrm{kcal} /$ day in accordance with scientific evidence, which has indicated the scant utility of therapies, which are strictly hypocaloric.

The proposed food plan, which deviates from the so-called "diabetic diet" imposed during the last decade on rigid parameters with regard to the intake of carbohydrates, has as its basic reference model the Mediterranean Diet. The delivered daily eating pattern provides a breakdown of macro-nutrients as detailed below.

The proportion of carbohydrates is about $55 \%$ of total daily energy input (range $45-60 \%$, and never less than $130 \mathrm{~g} /$ day), calibrated according to individual habits and the clinical condition of the subject, given that there is no scientific evidence which makes it possible to recommend the ideal amount of $\mathrm{CHO}$ for each diabetic patient.

As for fat intake, the dose foreseen in the diet does not exceed 35\% of total daily calories, with focus on a reduced supply of saturated and hydrogenated fats and an increase in the consumption of polyunsaturated fatty acids of type $\mathrm{W} 3$. The recommended protein requirement is $15 \%$ of the daily total, and the consumption of protein of vegetable origin is encouraged.

Additional recommendations include: an increase in the consumption of foods in the low GI bracket and an intake of fibre superior to $40 \mathrm{~g} /$ day or $20 \mathrm{~g} / 1000 \mathrm{kcal} /$ day, especially the soluble type derived from vegetable, fruits and pulses; a reduced intake of dietary sodium ( $2400 \mathrm{mg} /$ day, corresponding to $6 \mathrm{~g}$ of salt). It also advises against the consumption of sweeteners, alcohol, diet supplements and food products for diabetics. A reduction in the consumption of energydense foods, especially those rich in simple fats and sugars, is recommended.

Individual check-ups with the dietitian were scheduled after one month from the handover of the dietary plan, indicated in processing results as (T1), two months (T2) and six months (T3). Between T2 and $\mathrm{T} 3$, the subject received a telephonic reminder. At $\mathrm{T} 0$ and $\mathrm{T} 2$ the subject was given a food diary and was asked to compile it before the next check up (T1 and T3). During the check-ups the anthropometric parameters were assessed, the diary, food history and dietary trends were controlled and eventual doubts and questions discussed. The last clinical check-up (T3) made it possible to verify whether weight objectives had been achieved and if the difficulties encountered at the beginning of the programme had been resolved. On this occasion, blood chemistry indices were also evaluated and psychological tests regarding quality of life were re-administered.

For patients who did not take part in the food education project, but who followed exclusively the Group Care meetings, the investigated parameters were recorded at the beginning and at the end of the study.

The methods used for the assessment of eating habits are the food diary and $24 \mathrm{~h}$ Dietary Recall. The food diary is the most precise and accurate method for recording the food consumption of an individual [9].

It records the type and quantity of food, the time and the place of consumption. In the version used in the project, patients were asked to note down eventual negative feelings associated with meals (anger, boredom, sadness, etc.,) and any physical activity (of what type and for how long). Each patient was asked to compile the food diary in the 5 days leading up to the receipt of the diet and the 5 days preceding the check-up.

The 24-hour dietary recall ( $-24 \mathrm{HR})$ is a tool used to determine the average intake of nutrients over 24 hours. The collection was created by the dietitian to help the individual to quantify, as correctly as possible, the food and beverages consumed, including the most obvious or easily forgotten. It is a simple, straightforward tool, which allows an accurate and precise evaluation of the individual's real food consumption [10].

In the project this tool was used in all the individual meetings with the dietitian, with the aim of assessing food consumption with the help of the photographic food atlas. The time required for the collection of information was, on average, 20-30 minutes.

At the beginning and end of the project each subject had an interview with a qualified psychotherapist. Physical activity was recommended to all 28 patients, with a description of the benefits that this can bring for personal health. Motivation was reinforced when needed.

Table 1 illustrates the intervention plan. Blood chemistry parameters (HbAlc, fasting blood glucose, total cholesterol, HDL cholesterol, triglycerides, calculated LDL cholesterol) were measured at the beginning of the study and after six months of follow-up. Simultaneously, the psychotherapist carried out neuropsychological tests for the assessment of anxiety (SAS self-assessment scale - Self-Rating Anxiety Scale), depression (SDS self-assessment scale - Self-rating Depression Scale) and adaptation to diabetes (ATT19 - Diabetes Attitude Questionnaire) [11,12]. Any physical activity was recorded and evaluated in terms of frequency (days per week) both at the beginning of the study and after six months. A control group continued with Group Care with neither nutrition education imputs nor the prescription of a personalized dietary plan. There were frequent outpatient controls at brief intervals, in addition to diabetes routine care visits. The plan of intervention is shown in Table 1.

The aim of the study is to determine whether the referred patients, with personalized dietary support and regular individual check-up, compared to those patients followed exclusively by traditional educational Group Care methods, are able to achieve improvements with regard to anthropometric indices (the target goal being a 5\% reduction in body weight and a $5 \%$ reduction in waist circumference), blood chemistry indices (glycemic decompensation and lipid profile) and quality of life. 
Table 1. Plan of individualized intervention.

Presentation and explanation of the project to each subject, consent collection

First individual meeting with the dietician (60-90 minutes):

- recording of anthropometric indices (weight, waist circumference)

- evaluation of body composition, BMI calculation

- personal food history

- blood tests (HbAlc fasting glucose, total cholesterol, HDL cholesterol, triglycerides, calculated LDL cholesterol)

Second individual meeting with the dietician (after 2 days) - T0:

- delivery of the elaborated dietary plan, detailed explanation of the diet, nutrition education (eg. the strategies to be applied in case of emotional hunger, specific dietary recommendations for the disease, etc.)

- discussion of calculated anthropometric goals

- counseling and practical hints on practising physical activity

- neuro-psychological tests, carried out by qualified psychologist, for the assessment of anxiety (SAS self-assessment scale), depression (SDS self-assessment scale) and adaptation to diabetes (ATT19)

- request for the compilation of the food diary

1-month check up (T1):

- reassessment of anthropometric parameters (weight, waist circumference)

- control of food diary, food history and dietary trends

- counseling

\section{2-month check up (T2):}

- reassessment of anthropometric parameters (weight, waist circumference)

- control of food diary, food history and dietary trends

- counseling

- request for the compilation of the food diary

\section{Telephonic reminder}

\section{6-month check up (T3):}

- reassessment of anthropometric parameters (weight, waist circumference)

- counseling on the resolution of initial problems

- carry out control blood tests (HbAlc. fasting glucose, total cholesterol, HDL cholesterol, triglycerides, calculated LDL cholesterol)

- neuro-psychological tests for reassessment (SAS, SDS, ATT-19)

HbAlc: glycated hemoglobina; HDL: high-density lipoprotein: LDL: low-density lipoprotein; SAS: Self-rating Anxiety Scale; SDS: Self-rating Depression Scale; ATT19: Diabetes Attitude Questionnaire.

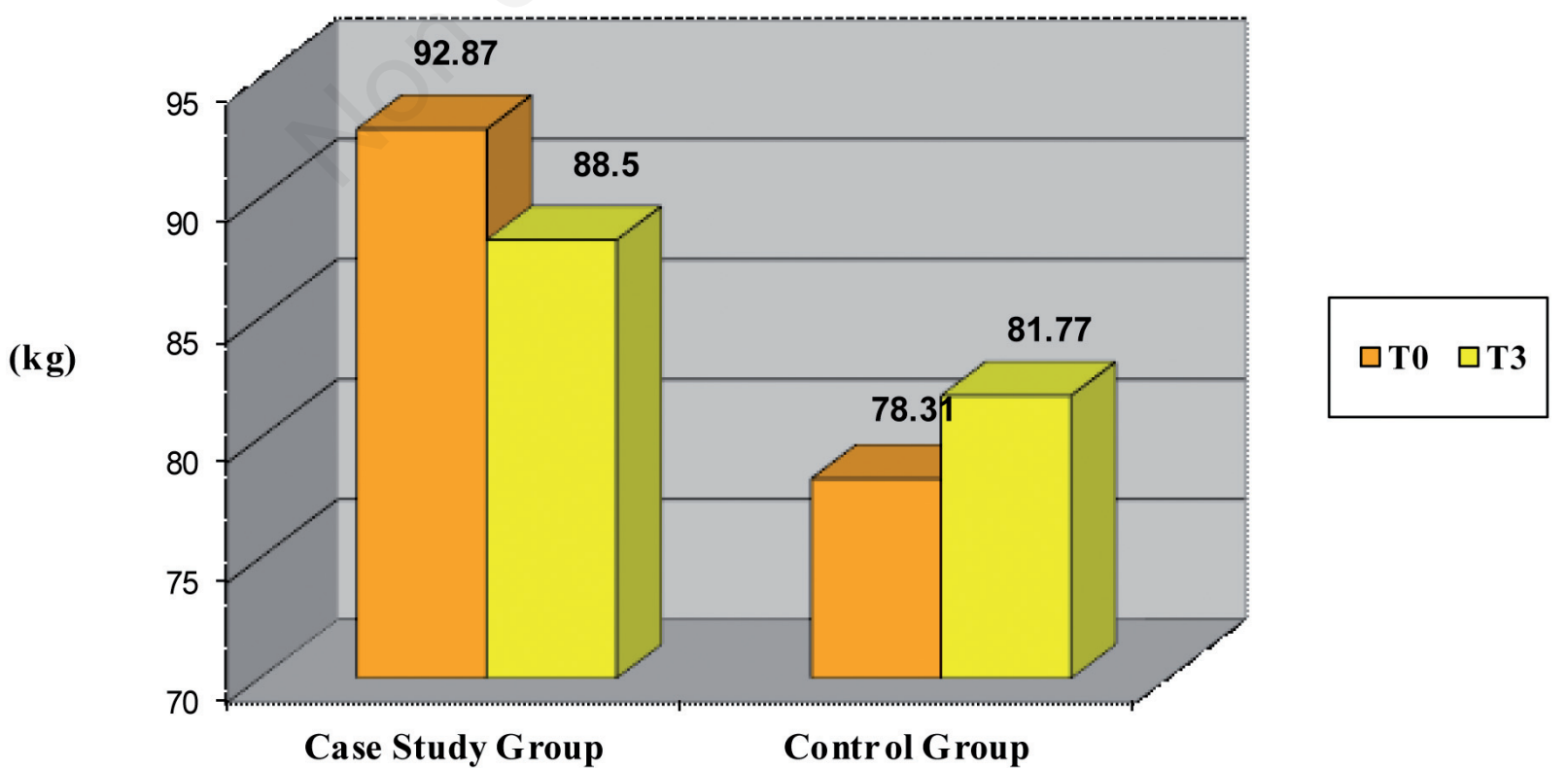

Figure 1. Weight variation; comparison between case study group and control group $(\mathrm{p}<0.05)$. 


\section{Statistical analysis}

Data are presented as median \pm SD. To highlight the differences among the various parameters, both within same sample and between different samples, for continuous variables the Student $t$-test for paired samples and for independent samples was used. As for the ordinal variables, the Wilcoxon test for paired samples was used.

To highlight any significant differences of the individual parameters at different times the method of analysis of variance Two-way repeated measures (ANOVA for repeated measures) was used. A statistical significance level of $p<0.05$ was taken into account.

The programme MedCalc ver. 14.0.1 was used in all the statistical analyses.

\section{Results}

Twelve women and sixteen men, between the ages of 36 and 73 , were recluted; each group was made up of 14 individuals, 8 men and 6 women. No individual dropped out. The average age of the subjects in the case study group was 61 and in the control group the average age was 63 . The average age of diagnosis was 48 in the case study group and 47 in the control group. As regards co-morbidity, often associated with diabetes, in the study population 23 subjects (11 in the case study and 12 among the control group) suffer from hypertension. Most of the subjects in the sample groups (78.6\% in the case study and $85.7 \%$ in the control group) are following medical therapy with oral hypoglycemic agents; no patient follows a regime of mixed therapy (oral hypoglycemic agents and insulin therapy).

The general characteristics of the sample groups are summarized in Table 2.

\section{Anthropometric indices}

In subjects involved in the personalized nutrition plan, body weight, on average, gradually decreases, as does the waist circumference, on the contrary to what happens in the control group.

In the case study group, mean weight loss was $4.62 \mathrm{~kg}$ in six months: the average starting weight was $92.87 \mathrm{~kg}$, which fell to $88.25 \mathrm{~kg}$ in six months (Figure 2). However, in the control group an average increase of $3.46 \mathrm{~kg}$ was detected over the six months (from $78.31 \mathrm{~kg}$ to $81.77 \mathrm{~kg}$ ) (Figure 1).

Table 2. Personal characteristics of subjects at the beginning of the study ( $\mathrm{p}$-valure: not significant).

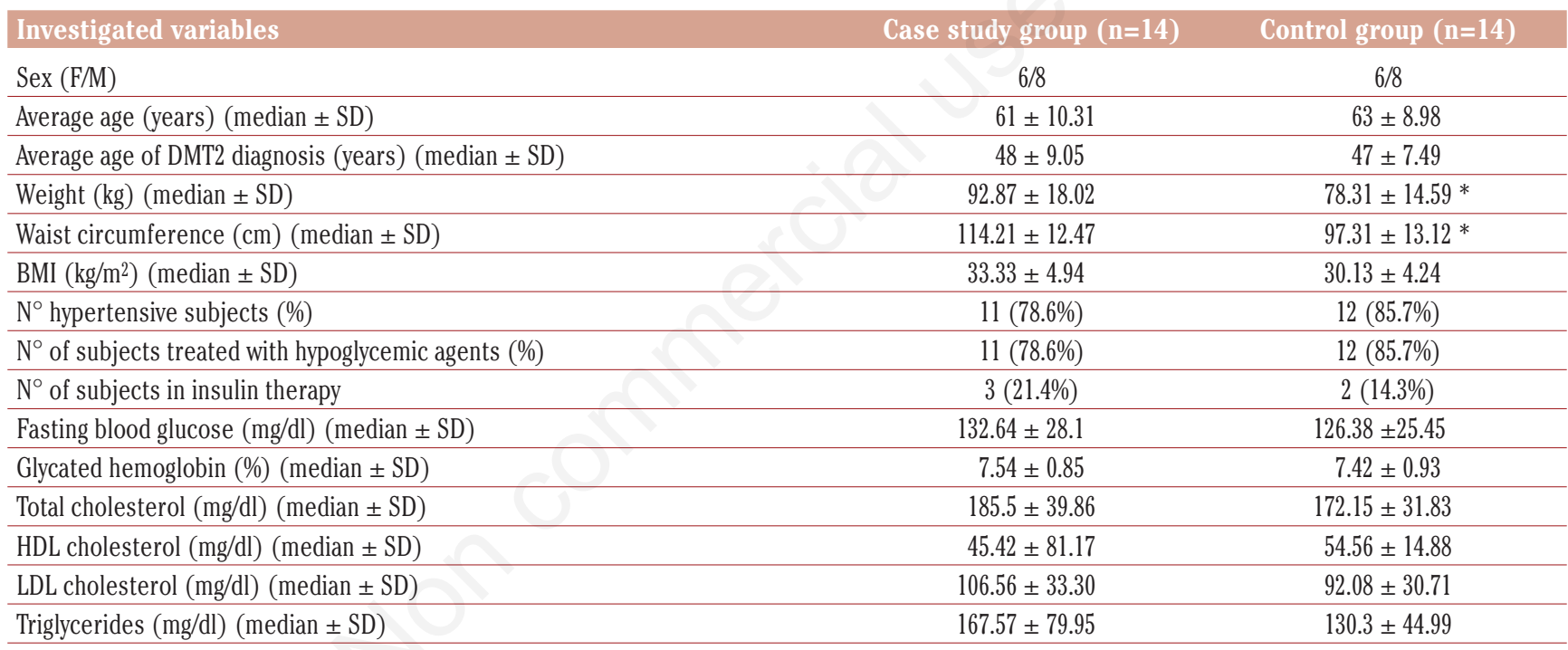

BMI: body mass index; HDL: high-density lipoprotein: LDL: low-density lipoprotein; ${ }^{*} \mathrm{p}<0.05$.

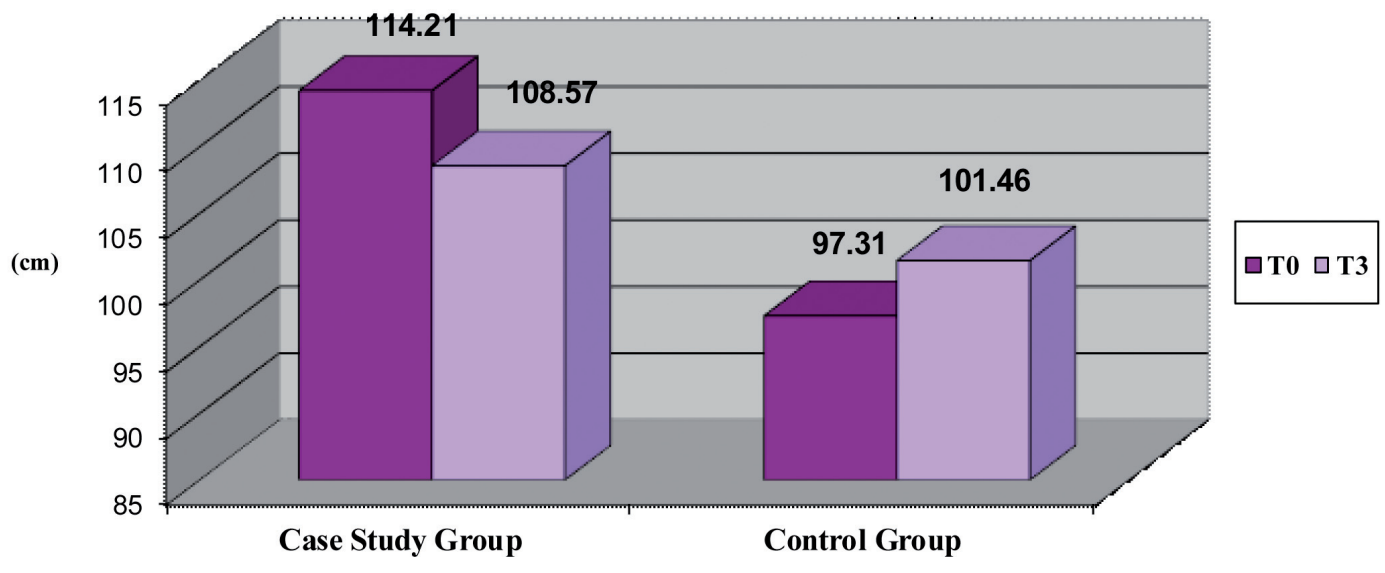

Figure 2. Waist circumference variation; comparison between case study group and control group $(\mathrm{p}<0.05)$. 
Regarding the waist circumference, an average decrease of $5.64 \mathrm{~cm}$ over six months in the study case group is recorded (from $114.21 \mathrm{~cm}$ to $108.57 \mathrm{~cm}$ at follow-up) (Figure 2). On the contrary, in the control group, this variable passes from an average of $97.31 \mathrm{~cm}$ to $101.46 \mathrm{~cm}$ in six months, with a significant $4.15 \mathrm{~cm}$ increase $(\mathrm{p}=0.0001)$ (Figure 2).

The statistical analysis carried out verified how weight loss and decrease in waist circumference are associated and occur simultaneously: in fact, fat distribution around the abdomen varies with weight change and with them the BMI value. In the control group, BMI increases from $30.13 \mathrm{~kg} / \mathrm{m}^{2}$ to $\mathrm{T} 0$ to $31.18 \mathrm{~kg} / \mathrm{m}^{2}$ at T3; an increase in the BMI value of 1.05 points $(\mathrm{p}=0.0011)$ is observed. In the case study group, instead, a reduction in BMI is witnessed, passing, in fact, in six months, from $33.33 \mathrm{~kg} / \mathrm{m}^{2}$ to $31.72 \mathrm{~kg} / \mathrm{m}^{2}$, with a drop of 1.6 points.

\section{Blood chemistry indices}

In the case study group, the fasting blood glucose level average at the beginning of the project was recorded at $132.64 \mathrm{mg} / \mathrm{dl}$, and after six months there was a decrease, with an average value of 117.23 $\mathrm{mg} / \mathrm{dl}$. The result is significant $(\mathrm{p}=0.0011)$ (Table 3 ). In the control group, however, the average value of fasting blood glucose was 126.38 $\mathrm{mg} / \mathrm{dl}$ at $\mathrm{T} 0$ and rose to $143.92 \mathrm{mg} / \mathrm{dl}$ after six months $(\mathrm{p}=0.0018)$ (Table 3).

Regarding glycated hemoglobin values, in the case study group a reduction from $7.54 \%$ to $6.87 \%$ after six months is observed, while the control group showed instead a $7.42 \%$ increase, up to $7.99 \%$ (Figure 3 ).

Table 3. Variation between T0 and T3 (6 months) of the clinical characteristics of the study participants, divided between case study and control groups.

\begin{tabular}{|c|c|c|c|c|c|c|}
\hline & \multicolumn{3}{|c|}{ Case study } & \multicolumn{3}{|c|}{ Control } \\
\hline & T0 & T3 & p-value & T0 & T3 & p-value \\
\hline Weight (kg) & $92.87 \pm 18.02$ & $88.25 \pm 16.2$ & I & $78.31 \pm 13.45$ & $81.77 \pm 13.82$ & $\mathrm{p}=0.0005$ \\
\hline Waist circumference $(\mathrm{cm})$ & $114.21 \pm 12.47$ & $108.57 \pm 16.2$ & / & $97.31 \pm 12.69$ & $101.46 \pm 13.36$ & $\mathrm{p}=0.0001$ \\
\hline $\operatorname{BMI}\left(\mathrm{kg} / \mathrm{m}^{2}\right)$ & $33.33 \pm 4.94$ & $31.72 \pm 4.51$ & / & $30.13 \pm 4.08$ & $31.18 \pm 4.34$ & $\mathrm{p}=0.0011$ \\
\hline Fasting blood glucose (mg/dl) & $132.64 \pm 28.1$ & $117.23 \pm 1.16$ & 0.0011 & $126.38 \pm 25.45$ & $143.92 \pm 20.96$ & $\mathrm{p}=0.0018$ \\
\hline Glycated hemoglobin (\%) & $7.54 \pm 0.85$ & $6.87 \pm 0.78$ & 0.0074 & $7.42 \pm 0.93$ & $7.99 \pm 0.90$ & $\mathrm{p}=0.0101$ \\
\hline Total cholesterol (mg/dl) & $185.5 \pm 39.86$ & $167.5 \pm 31.87$ & 0.0005 & $172.15 \pm 31.83$ & $185 \pm 33.89$ & $\mathrm{p}=\mathrm{NS}$ \\
\hline HDL $(\mathrm{mg} / \mathrm{dl})$ & $45.42 \pm 81.17$ & $52.79 \pm 0.26$ & 0.003 & $54.56 \pm 14.88$ & $46.76 \pm 8.53$ & 0.007 \\
\hline LDL (mg/dl) & $106.56 \pm 33.30$ & $87.76 \pm 30.56$ & 0.006 & $92.08 \pm 30.71$ & $112.01 \pm 26.06$ & 0.009 \\
\hline Triglycerides (mg/dl) & $167.57 \pm 79.95$ & $135.14 \pm 52.66$ & 0.05 & $130.3 \pm 44.99$ & $160 \pm 46.28$ & 0.003 \\
\hline
\end{tabular}

BMI: body mass index; HDL: high-density lipoprotein: LDL: low-density lipoprotein; NS: not significant.

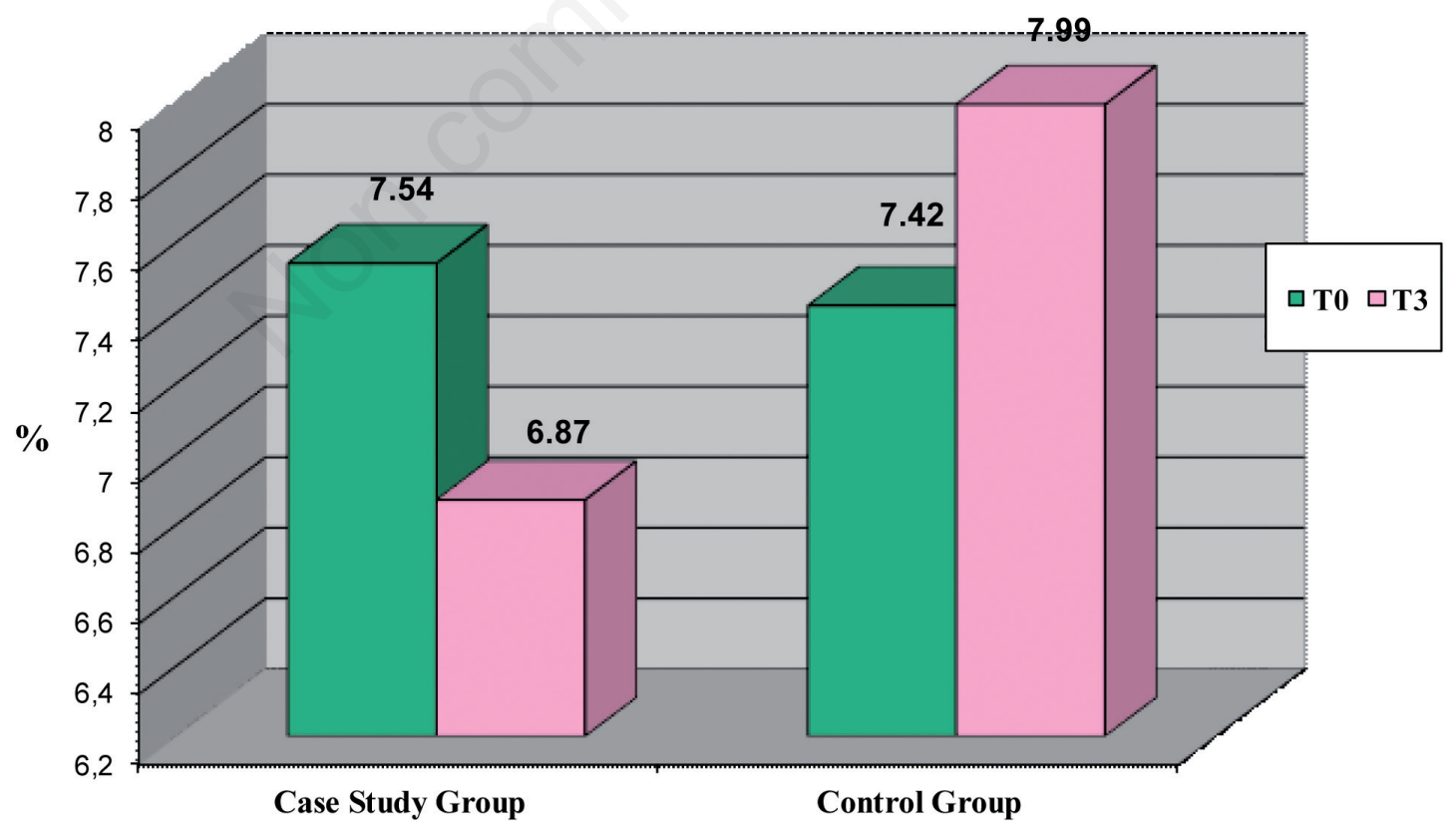

$\mathbf{p}<\mathbf{0 . 0 1}$

$\mathbf{p}=\mathbf{0 . 0 1}$

Figure 3. Glycated hemoglobin variation between T0 and T3; comparison between case study group and control group. 
Significant variations between the two groups were also detected for the lipid profile, as shown in Table 3: in the case study group, total cholesterol values decreased (even if it is not significative), while in the control group a trend towards an increase in total cholesterol is observed. Analagously, in the case study group a reduction in LDL cholesterol and triglycerides and an increase in HDL cholesterol is seen, while in the changes in the control group the trend moves in the opposite direction.

Even in regard to food changes monitored through the food diary, in the case study group, the excessive consumption of sweets and fruit more than halved over six months, the intake of bread and pasta was controlled, with the introduction of whole-grain products; all the subjects introduced a daily consumption of two servings of vegetables.

\section{Physical activity}

The control group shows a reduction in the frequency of physical activity, although no statistical relevance is reached: if at the beginning of the study the median is 2 sessions / week for at least 50 minutes (with heterogeneity in practice, from subjects who never do exercise to the person exercising 3 times a week), after six months, the median value drops to 1 session: the highest level of physical activity is practiced, in fact, twice a week. This means that half of the subjects are active for between $0 / 1$ session per week (with some subjects who interrupted all physical activity), whereas the other half is involved in physical activity for $1 / 2$ sessions per week.

On the contrary, the study group registers a reverse tendency. At the beginning the median value is 0.5 sessions per week: half of the subjects do no physical activity while the other half practices sport from 13 times a week. After six months, there has been a turnaround, given that the median value becomes 3 (sessions) $(\mathrm{p}<0.01)$. The minimum value is 1 , and this means that half of the subjects in this group carry out physical activity from 1 to 3 times a week, while the remainder exercise with a frequency equal to 3 (or more) times a week. The variation is represented in Figure 4.

\section{Evaluation of the quality of life}

In the case study group, it is possible to observe how, at the beginning of the study, out of a total of 14 subjects, 5 presented an adaptation to diabetes at a medium level; there was a low level of depression in 2 individuals, while mild anxiety was observed in 1 subject. The patient with anxiety also presented a medium adaptation to diabetes; the total number of subjects with some type of psychological difficulty in this group were, at that time, 6 out of 14 (43\%). The psychological study was conducted again at the end of six months and revealed the absence of disorders of an anxious or depressive type and a good adjustment to diabetes (Figure 5). In particular, the average value of ATT-19 rose to 78 (average value at $\mathrm{T} 0=70.3$ ).

The psychological tests conducted in the control group in the early stages of the study showed that there was medium adaptation to diabetes in 4 subjects, while a state of mild depression was identified in 2 subjects; 1 of them also showed a slight degree of anxiety. The subjects of this group with psychological problems were, in total, 6 out of 14 (43\%).

When the second test was administered, six months later, it was possible to notice a worsening of these values: those with medium adaptation to diabetes were now 6 and, in addition, a case of inadequate adaptation in one individual was registered; those with mild depression were now 3 , while the mild state of anxiety present in one of the subjects at $\mathrm{T} 0$ remained (in coexistence with a depressive state) (Figure 6). In total, those with psychological problems are 9 out of 14 (64\% of the sample).

\section{Discussion}

This study demonstrates that in diabetic patients, who are overweight with sub-optimal glycometabolic compensation, nutritional counseling with checks and reinforcements can achieve a significant reduction in body weight. Better glycometabolic compensation in the course of the 6-month study was achieved.

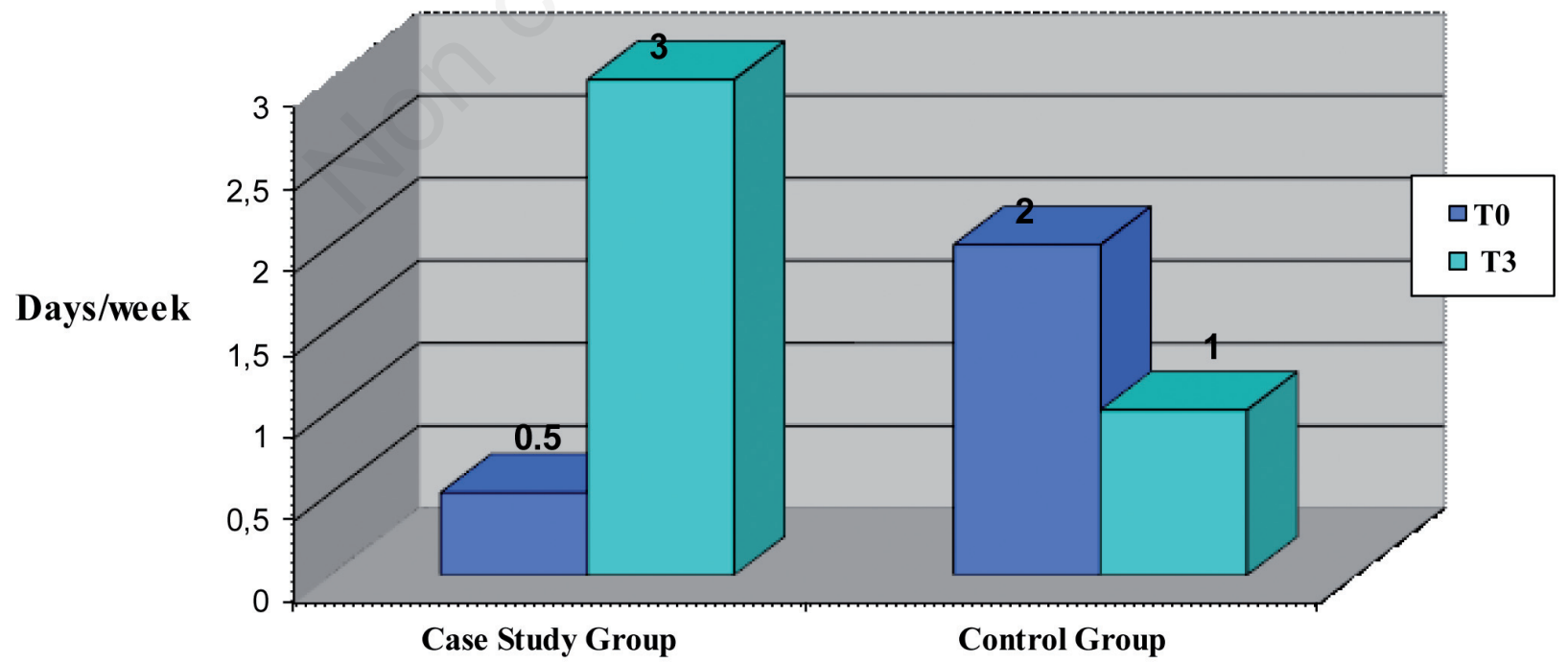

$\mathbf{p}<\mathbf{0 . 0 1}$

$\mathbf{p}=\mathbf{N S}$

Figure 4. Physical activity variation between T0 and T3; comparison between case study group and control group. 


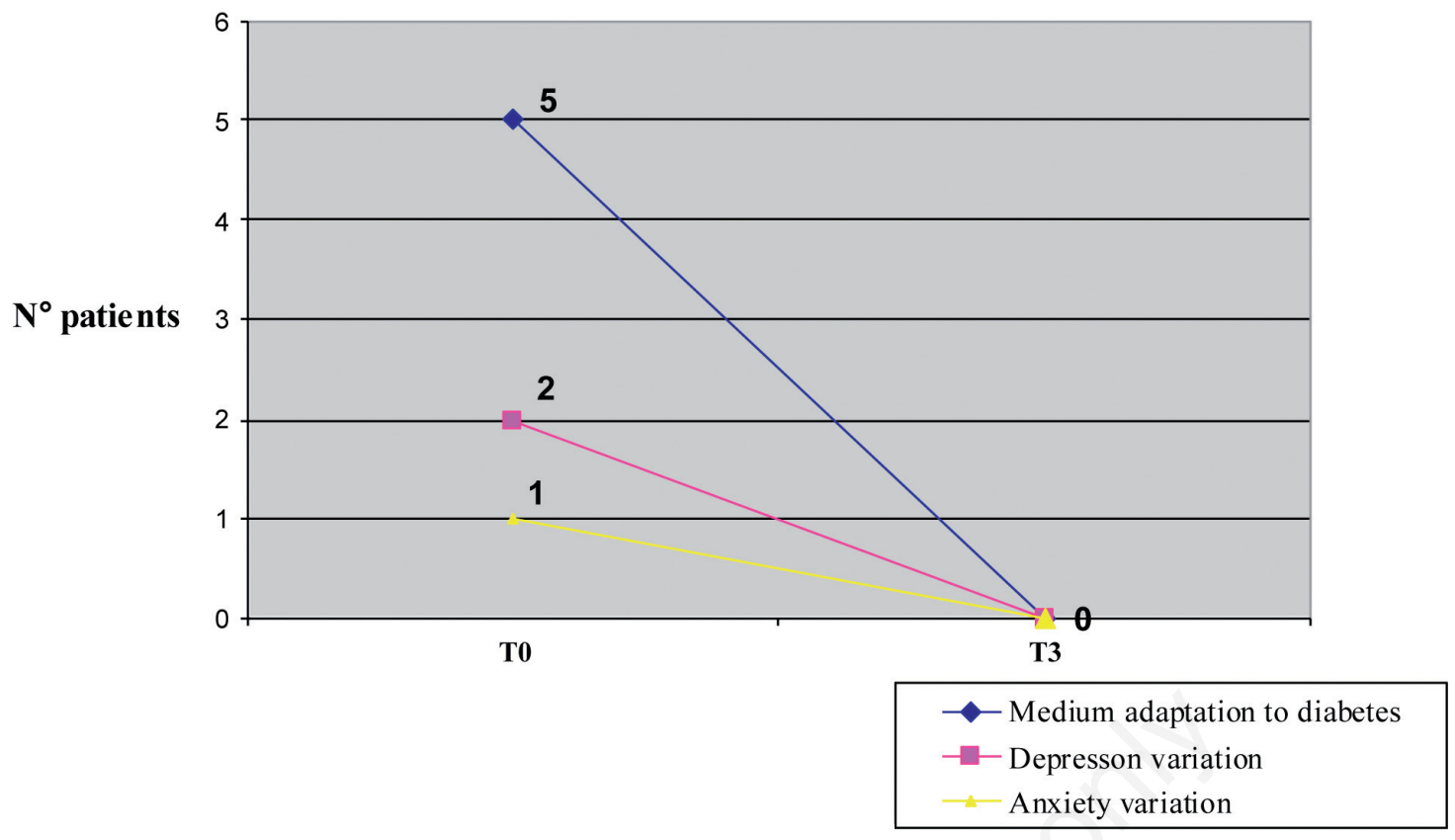

Figure 5. Psychological assessment in case study group: number of patients with medium adaptation to diabetes on the ATT19 scale, depression and anxiety; variations between $\mathrm{T} 0$ and $\mathrm{T} 3$.

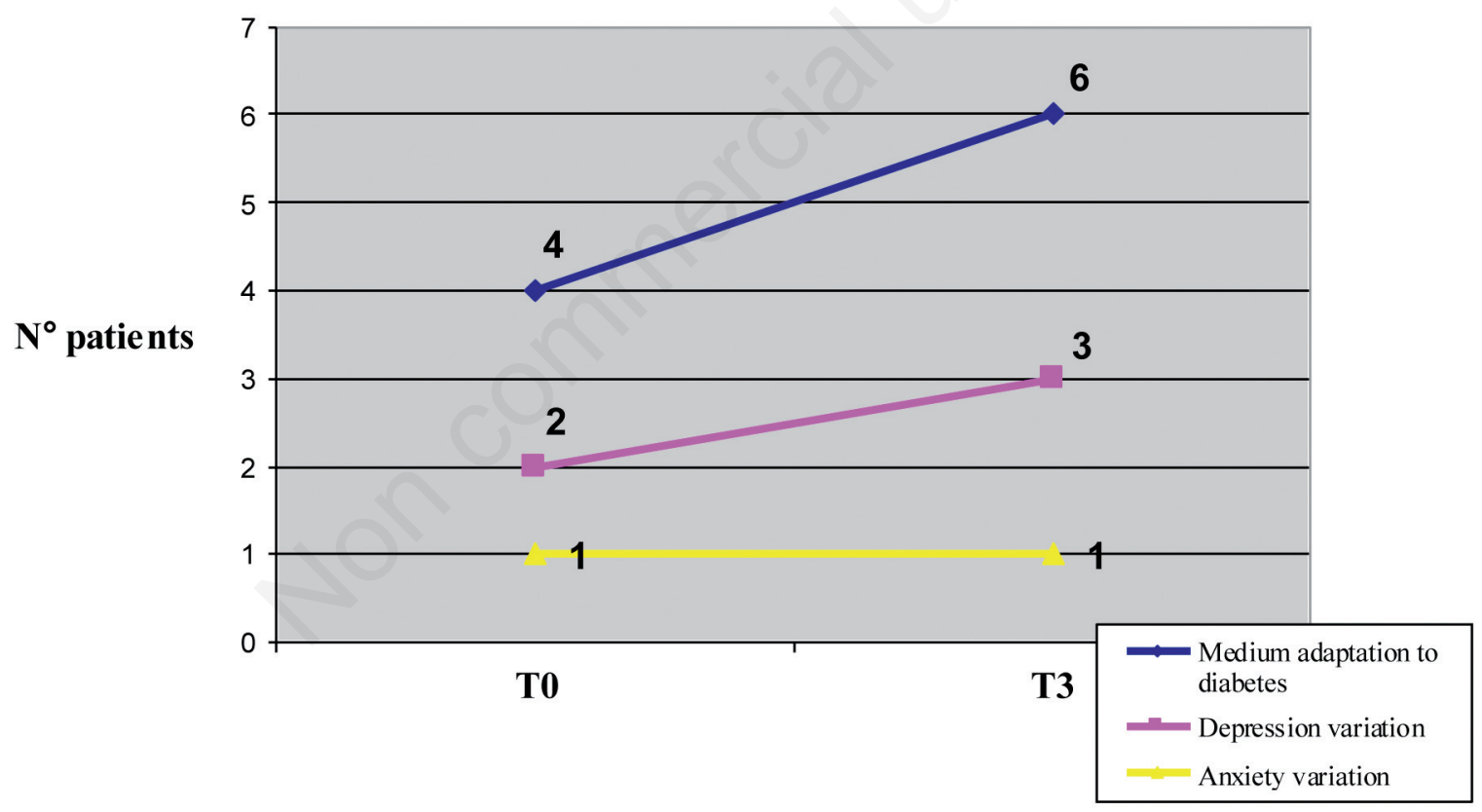

Figure 6. Psychological assessment in control group: number of patients with medium adaptation to diabetes on the ATT19 scale, depression and anxiety; variations between $\mathrm{T} 0$ and $\mathrm{T} 3$.

The Italian Barometer Diabetes Report 2013 [13] highlights how the timely diagnosis and the constant control of people with diabetes, thanks to quality therapies, can reduce by $10-25 \%$ the risk of early complications (retinopathy and diabetic nephropathy) and by $15-55 \%$ the risk of advanced complications (chronic renal failure, coronary artery disease, loss of vision). In addition, it is estimated that these actions are able to delay the onset of complications by more than five years and to prolong the lives of people with T2DM. In the long term, a similar im- provement of the therapeutic framework will allow for an average reduction of more than $30 \%$ in costs.

The average reduction over six months was $4.97 \%$ in body weight $(-4.61 \mathrm{~kg}), 4.94 \%$ in waist circumference $(-5.64 \mathrm{~cm})$ and $4.8 \%$ in BMI ( -1.61 points). In the Look AHEAD study, whose purpose was to act on the lifestyle of individuals with T2DM, it showed that intensive treatment through diet and increased physical activity can induce clinically significant changes in weight loss and waist circumference reduction 
in overweight and obese patients suffering from T2DM, with maintenance of the achieved weight loss at a distance of 4 years (which turned out to be $>5 \%$ ) in more than $45 \%$ of the sample investigated ( $-8.6 \%$ in the study group $v s-0.7 \%$ in the control group) [14,15].

The effectiveness of intervention in the lifestyle of patients with T2DM was also highlighted by a meta-analysis of 2014, which noted that, on average, interventions in lifestyle have shown a statistically significant reduction in weight, with an average loss of about $3.3 \%$ of starting weight [16].

Obviously, the illustrated results have limitations: the average weight remains high, waist circumference is greater than the values recommended by the International Diabetes Federation and the World Health Organization (WHO) and the BMI falls more within the range of obesity (approaching, however, the "overweight" cut off). However, the observed changes seem promising: the pre-established weight loss targets, set during drafting, were achieved, and even if the values of anthropometric parameters investigated are still high, the desired loss in accordance with the literature and Guidelines (5\% reduction) was recorded [17].

Moreover, fasting glycaemia levels also seem to feel the effects of personalized dietetic intervention ( $\mathrm{p}<0.05)$. The study Emerging Risk Factors Collaboration has established the risk of death from all causes and from specific causes (with emphasis on cardiovascular diseases) in a population of 698,782 individuals enrolled in 102 prospective studies in which they were registered and 52,765 fatal and non-fatal cardiovascular events $[18,19]$. Diabetes conferred twice the risk of vascular disease and its effect was also demonstrated when the analysis was adjusted for all other conventional cardiovascular risk factors. It also emerged that an increased plasma glucose concentration $>100 \mathrm{mg} / \mathrm{dl}$, and in any case under the limits for the diagnosis of diabetes mellitus, is associated with an increased risk of cardiovascular diseases, supporting the hypothesis that hyperglycemia (or some element closely related to it) is an independent risk factor. This view is also supported by the fact that, in the same study, the risk of death from diabetes is markedly attenuated when the relationship is monitored for blood glucose values. Guidelines agree that the treatment of diabetes should be tailored to each patient to achieve stable HbAlc values below 7.0\%, which helps to prevent the incidence and progression of complications [20]. However, the importance of the personalization of glycemic goals must be noted: for non-elderly patients or patients without comorbidities that make them particularly fragile, the value for HbAlc to aim for is $6.5 \%$ [20]. From the tests carried out it was possible to detect, in the case study group, an HbAlc decrease of $-8.8 \%$ ( -0.67 from 7.54 to 6.87 in six months), while in the control group the increment detected was of $7.13 \%$ (from 7.42 to 7.99 ). It is possible to assume that it is also due to the change in eating habits (good control in the intake of simple sugars and complex carbohydrates was, in fact, verified) and increased physical activity which, as reported in the literature, favours the improvement in glycemic compensation. On the other hand, it has to be said that all the patients are under medication and therefore it is possible that the observed results are not exclusively attributable to weight loss and the change in lifestyle. However, the overall therapeutic target indicated for patients with T2DM (HbAlc $<7 \%)$ was achieved, given that the average value at $\mathrm{T} 3$ was $6.87 \%$.

The UKPDS study observed how for every 1-percentage point decrease in glycated hemoglobin it is possible to significantly reduce the risk of serious complications [20]. The study shows, in fact, how the effects of good glycemic control will result in a significant reduction in mortality and of micro and macrovascular complications (for example, there was a reduction of $14 \%$ in the risk of heart attacks and of $37 \%$ in microvascular complications).

The lipid profile is clearly favorably impacted by the personalized diet and total cholesterol is reduced $(-9.7 \% ; \mathrm{p}<0.05)$ while increasing in the control group $(6.9 \%, \mathrm{p}=\mathrm{ns})$. A similar argument can be made for HDL cholesterol, an increase in which was found to be inversely associated with cardiovascular disease and, as confirmed by the scientific evidence, it appears to be directly correlated to the frequency of sporting activities [20]. In the case study group, HDL cholesterol increased, in fact, by $13.96 \%$ (parallel to the increase in physical activity), while in the control group there was a reduction in this value $(-14 \%)$, as well as in the frequency with which sport was practised, which was halved.

The changes relative to LDL cholesterol are also interesting for the difference observed in the two groups: if in the case study group there was a decrease of $17.6 \%$, the control group revealed an increase of $17.8 \%$. The reverse trend observed in both groups is also confirmed by the average values of triglycerides: a decrease of $19.35 \%$ in the case study group and an increase of $22.8 \%$ in the control group. The Look AHEAD study showed that a weight reduction of $>5 \%$ is associated with increased HDL cholesterol $(+5 \mathrm{mg} / \mathrm{dl})$ and reduced triglycerides $(-40$ $\mathrm{mg} / \mathrm{dl})[21]$.

Clinical and scientific evidence to support the importance of treating all the factors contributing to increased cardiovascular risk comes from the study STENO 2: the more aggressive therapeutic approach on all fronts (diet, exercise, medication, etc.) made more inroads for improvement in all vascular complications, both for small and for large arteries, than more traditional intervention [22].

The scientific literature and Guide Lines agree about the importance of sporting activities for people with T2DM, for the improvement in glycemic compensation and reduction of insulin-resistance; it also encourages weight loss and helps maintain it, as well as having a favorable impact on the pressure tone and mood $[23,24]$.

The result obtained in our trial seems promising: all subjects perform exercise at least once a week and the median value has more than doubled; furthermore, the increase remained constant over the six months.

Furthermore, the case study group seems to have received benefits also with regard to the perceived quality of life. Scientific evidence agrees that individual psycho-educational intervention is essential in order to achieve results in the long term [25]. Many scientific studies based on educational projects aimed at increasing adaptation to diabetes strategies and ameliorating the symptoms of the disease, showed improvement in many indicators of quality of life, such as self-esteem, depression, anxiety, coping strategies and emotional well-being.

Finally, it was found that diabetes self-management and education (DSME) is linked to an increase in knowledge about the disease, reduction in HbAlc and weight loss [26-29].

In conclusion, this case study/control group intervention brought to light how an individual path with regular check ups, the drafting of a personalized dietary plan and the inclusion of sustainable changes (including increased physical activity) in everyday life are strategic components for the achievement of objectives in a population of diabetics resistant to treatment through Group Care.

\section{Study limitations}

The major limitation is represented by the reduced number of the sample $(\mathrm{N}=28)$. However, the results obtained are promising, expressing, and even with a limited number of cases, a statistical significance. In addition, a period of time longer than six months could lead to different results and/or render the study more robust. It must be kept in mind that there may be other confounding factors or factors not considered. Studies are needed with a larger sample size, a more extended period of time, with a different statistical design (of force majeure, as in prospective studies). 


\section{References}

1. AAVV. L'educazione terapeutica nel paziente diabetico. In: Supplemento al Giornale Italiano di Diabetologia. Milano: Utet Periodici Scientifici; 1999; Vol. 19, n. 4.

2. ADA. Standards of medical care in diabetes - 2014. Diabetes Care 2014;37:S14-S80.

3. Rotella CM, Ricca V, Mannucci E. L'Obesità. Manuale per la diagnosi e la terapia. Firenze: SEE;1997.

4. AMD-SID. Standard italiani per la cura del diabete mellito 2014.

5. ADI-AMD-SID - Raccomandazioni 2013-2014: La terapia medica nutrizionale nel diabete mellito.

6. Trento M, Passera P, Borgo E et al. A 5 year randomized controlled study of learning, problem solving ability, and quality of life modifications in people with type 2 diabetes managed by group care. Diabetes Care 2004;27:670-5.

7. Trento M, Passera P, Bajardi M et al. Lifestyle intervention by group care prevents deterioration of type 2 diabetes: a 4 year randomized controlled clinical trial. Diabetologia 2002;45:1231-9.

8. Trento M, Tomelini M, Basile M et al. Il modello assistenziale della Group Care ed i corsi residenziali su: Management del diabete tipo 2 mediante Group Care del Dip. di Medicina interna, Laboratorio di Pedagogia Clinica, Università di Torino. Giornale Italiano di Diabetologia e Metabolismo 2007;27:47-53.

9. Bedogni G, Cecchetto G. Manuale ANDID di valutazione dello stato nutrizionale. Seu Editrice; 2009.

10. Binetti P, Marcelli M, Baisi R. Manuale di nutrizione clinica e scienze dietetiche applicate. Roma: Seu Editrice; 2005.

11. Zung WWK. A self-rating depression scale. Arch Gen Psychiatry 1965;12:63-70.

12. Welch G, Dunn SM, Beeney LJ. The ATT39: a measure of psychological adjustment to diabetes. In: Bradley C, ed. Handbook of Psychology and Diabetes. Harwood Academic Publishers; Switzerland: 1994, pp 223-46.

13. Italian Barometer Diabetes Report 2013 - L'impatto del diabete in Italia.

14. Look AHEAD Research Group. Cardiovascular effects of intensive lifestyle intervention in type 2 diabetes. N Engl J Med 2013;369:145-54.

15. Wadden TA, Neiberg RH, Wing RR, et al. Four-year weight losses in the look AHEAD study: factors associated with long-term success. Obesity 2011;19:1987-98.

16. Terranova CO, Brakenridge CL, Lawler SP, et al. Effectiveness of lifestyle-based weight loss interventions for adults with type 2 dia- betes: a systematic review and meta-analysis. Diabetes Obes Metab 2015;17:371-8.

17. Nonino F, Vignatelli L, Magrini N. Linee guida sulla gestione del diabete mellito di tipo 2. Sistema nazionale di linee guida-Istituto Superiore di Sanità; 2008.

18. Emerging Risk Factors Collaboration, Sarwar N, Gao P, et al. Diabetes mellitus, fasting blood glucose concentration, and risk of vascular disease: a collaborative meta-analysis of 102 prospective studies. Lancet 2010;375:2215-22.

19. Emerging Risk Factors Collaboration, Seshasai SR, Kaptoge S, et al. Diabetes mellitus, fasting glucose, and risk of cause-specific death. N Engl J Med 2011;364:829-41.

20. [No authors listed]. Effect of intensive blood-glucose control with metformin on complications in overweight patients with type 2 diabetes (UKPDS 34). Lancet 1998;352:854-65. Erratum in Lancet 1998 Nov 7;352:1558.

21. Wing R, Lang W, Wadden TA, et al. Benefits of modest weight loss in improving cardiovascular risk factors in overweight and obese individuals with type 2 Diabetes. Diabetes Care 2011;34:1481-6.

22. Gaede P, Lund-Andersen H, Parving HH, et al. Effect of a multifactorial intervention on mortality in type 2 diabetes. $\mathrm{N}$ Engl $\mathrm{J}$ Med 2008;358:580-91.

23. Dunstan DW, Daly RM, Owen N, et al. High-intensity resistance training improves glycemic control in older patients with type $2 \mathrm{di}$ abetes. Diabetes Care 2002;25:1729-36.

24. Castaneda C, Layne JE, Munoz Orians L, et al. A randomized controlled trial of resistance exercise training to improve glycemic control in older adults with type 2 diabetes. Diabetes Care 2002;25:2335-41.

25. Ciaccio S, Valentini U. Il ruolo dell'educazione terapeutica nella cronicità. MeDia 2011; 11:139-144.

26. Inzucchi SE, Bergenstal RM, Buse JB, et al. Management of hyperglycemia in type 2 diabetes: a patient-centered approach. Position statement of the American Diabetes Association (ADA) and the European Association for the Study of Diabetes (EASD). Diabetes Care 2012;35:1364-79.

27. Gary TL, Genkinger JM, Guallar E, et al. Metanalysis of randomized educational and behavioral interventions in type 2 diabetes. Diabetes Educ 2003;29:488-501.

28. AADE. Diabetes inpatient management. Diabetes Educator 2012; 38:142-6.

29. Rubin RR, Peyrot M, Saudek CD. The effect of a diabetes education program incorporating coping skills training on emotional wellbeing and diabetes self-efficacy. Diabetes Educator 1993;19:210-4. 